Article

\title{
Probing Dynamics in Colloidal Crystals with Pump-Probe Experiments at LCLS: Methodology and Analysis
}

\author{
Nastasia Mukharamova ${ }^{1}$, Sergey Lazarev ${ }^{1,2}$, Janne-Mieke Meijer ${ }^{3,9}{ }^{\text {, Matthieu Chollet }}{ }^{4}$, \\ Andrej Singer ${ }^{5}$, Ruslan P. Kurta ${ }^{1,10}$, Dmitry Dzhigaev ${ }^{1}$, Oleg Yu. Gorobtsov ${ }^{1}$, \\ Garth Williams 4,11, Diling Zhu ${ }^{4}$, Yiping Feng ${ }^{4}$, Marcin Sikorski 4,10, Sanghoon Song ${ }^{4}$, \\ Anatoly G. Shabalin ${ }^{1,12}$, Tatiana Gurieva ${ }^{1}$, Elena A. Sulyanova ${ }^{6}$, Oleksandr M. Yefanov ${ }^{7}$ \\ and Ivan A. Vartanyants $1,8, *$ \\ 1 Deutsches Elektronen-Synchrotron DESY, Notkestrasse 85, D-22607 Hamburg, Germany; \\ nastasia.mukharamova@desy.de (N.M.); sergey.lazarev@desy.de (S.L.); ruslan.kurta@xfel.eu (R.P.K.); \\ dmitry.dzhigaev@desy.de (D.D.); oleg.gorobtsov@desy.de (O.Y.G.); a.shabalin.r@gmail.com (A.G.S.); \\ tatyana.guryeva@desy.de (T.G.) \\ 2 National Research Tomsk Polytechnic University (TPU), Pr. Lenina 30, 634050 Tomsk, Russia \\ 3 Van 't Hoff Laboratory for Physical and Colloid Chemistry, Debye Institute for Nanomaterials Science, \\ Utrecht University, Padualaan 8, 3508 TB Utrecht, The Netherlands; janne-mieke.meijer@uni-konstanz.de \\ 4 SLAC National Accelerator Laboratory, 2575 Sand Hill Rd, Menlo Park, CA 94025, USA; \\ mchollet@slac.Stanford.edu (M.C.); gwilliams@bnl.gov (G.W.); dlzhu@slac.stanford.edu (D.Z.); \\ yfeng@slac.stanford.edu (Y.F.); marcin.sikorski@xfel.eu (M.S.); sanghoon@slac.stanford.edu (S.S.) \\ 5 UC San Diego, 9500 Gilman Dr., La Jolla, CA 92093, USA; singer.andrej@gmail.com \\ 6 Shubnikov Institute of Crystallography RAS, Leninskii Pr. 59, 119333 Moscow, Russia; \\ sulyanova@gmail.com \\ 7 Center for Free-Electron Laser Science, DESY, Notkestraße 85, D-22607 Hamburg, Germany; \\ oleksandr.yefanov@cfel.de \\ 8 National Research Nuclear University MEPhI, Kashirskoye Ch. 31, 115409 Moscow, Russia \\ 9 Present address: Department of Physics, University of Konstanz, D-78457 Konstanz, Germany \\ 10 Present address: European XFEL GmbH, Holzkoppel 4, D-22869 Schenefeld, Germany \\ 11 Present address: NSLS-II, Brookhaven National Laboratory, Upton, NY 11973-5000, USA \\ 12 Present address: UC San Diego, 9500 Gilman Dr., La Jolla, CA 92093, USA \\ * Correspondence: ivan.vartaniants@desy.de; Tel.: +49-040-8998-2653
}

Academic Editor: Kiyoshi Ueda

Received: 4 April 2017; Accepted: 8 May 2017; Published: 19 May 2017

\begin{abstract}
We present results of the studies of dynamics in colloidal crystals performed by pump-probe experiments using an X-ray free-electron laser (XFEL). Colloidal crystals were pumped with an infrared laser at a wavelength of $800 \mathrm{~nm}$ with varying power and probed by XFEL pulses at an energy of $8 \mathrm{keV}$ with a time delay up to $1000 \mathrm{ps}$. The positions of the Bragg peaks, and their radial and azimuthal widths were analyzed as a function of the time delay. The spectral analysis of the data did not reveal significant enhancement of frequencies expected in this experiment. This allowed us to conclude that the amplitude of vibrational modes excited in colloidal crystals was less than the systematic error caused by the noise level.
\end{abstract}

Keywords: free-electron laser; pump-probe; colloidal crystal; dynamics

\section{Introduction}

Acoustic motion in nanoscale objects induced by light is of importance for both fundamental science and applications. This interest is due to various potential applications in acousto-optical devices 
which can be used for ultrafast manipulation and control of electromagnetic waves by hypersonic (GHz) acoustic waves [1]. Colloidal crystals, formed by the self-assembly method, have shown phononic band gaps in the $\mathrm{GHz}$ frequency range [2-7]. Due to the recent progress in the fabrication of high-quality colloids, this provides an opportunity to produce inexpensive phononic crystals.

Dynamics in submicrometer colloidal crystals was investigated using different techniques such as Raman scattering [8], Brillouin light scattering [2], and optical pump-probe spectroscopy [4-7]. However, these methods have limited spatial resolution and are not suitable for the observation of the detailed structure of the sample. Furthermore, for some materials obtaining a sufficient refractive index is a challenging task, if not impossible.

At the same time, newly-developed X-ray free-electron lasers (XFELs) produce X-rays with unprecedented brightness, pulse duration and coherence, which are ideal for performing time-resolved experiments on various materials with a time resolution that outperforms that of third-generation synchrotron sources [9-11]. Moreover, the femtosecond X-ray pulses from free-electron laser sources allow an investigation of transient states of time-dependent processes [12-17]. In this respect, XFELs are especially well suited for investigating ultrafast structural dynamics of colloidal crystals.

The vibrations of the colloidal crystals were recently measured at free-electron laser FLASH in Hamburg [18]. The polystyrene colloidal crystals were pumped by an infrared (IR) laser of $800 \mathrm{~nm}$ and probed by an X-ray pulse with a wavelength of $8 \mathrm{~nm}$. This pump-probe experiment indicated the possibility of observation of the dynamics in the colloidal crystals by diffraction with a FEL. To give a reliable interpretation of the observed results, it was necessary to investigate the dynamics with a larger time interval and better statistics.

Here we present the results of a time-resolved pump-probe diffraction experiment in which the ultrafast dynamics in colloidal crystals induced by an IR laser was investigated. A theoretical analysis of the vibrations of the colloidal crystals was performed, and the vibrational frequency was calculated. This analysis was applied to the experimental results.

\section{Experiment}

The pump-probe experiments were performed at the Linac Coherent Light Source (LCLS) [9] in Stanford, USA at the X-ray Pump Probe (XPP) beamline [19]. LCLS was operated in the Self Amplified Spontaneous Emission (SASE) mode [20]. A sketch of the experiment is shown in Figure 1. A double-crystal diamond (111) monochromator with the thicknesses of the monochromator crystals of $100 \mu \mathrm{m}$ and $300 \mu \mathrm{m}$, enabled splitting of the primary X-ray beam into a pink (transmitted) and a monochromatic (reflected) branches and simultaneous operation of two beamlines (see Figure 1) [21]. We employed the monochromatic regime of LCLS with the energy of a single XFEL pulse of $8 \mathrm{keV}$ $\left(1.5498 \AA\right.$ ), energy bandwidth of $4.3 \times 10^{-5}$ [22], and a pulse duration of about $50 \mathrm{fs}$ as provided by electron bunch measurements at a repetition rate of $120 \mathrm{~Hz}$.

The X-ray beam was focused using the compound refractive lenses (CRLs) on the sample down to $50 \mu \mathrm{m}$ full width at half maximum (FWHM). The photon flux of the beam at the sample position was about $10^{9} \mathrm{ph} /$ pulse. This allowed us to perform measurements in a non-destructive regime. In the experiment, the colloidal crystal was oriented vertically, with its surface perpendicular to the incoming XFEL pulse in the transmission diffraction geometry.

Colloidal crystal films were prepared using the vertical deposition method [23] from spherical colloids consisting of two different materials, either polystyrene (PS) or silica. The crystalline films consisted of 30-40 particle layers, depending on the position on the film along the growth direction (the number of crystal layers increases at later growth times). After crystal growth, some of the films were covered with a $20-50 \mathrm{~nm}$ thick aluminum layer to increase interaction with the IR laser. In total, three types of samples were investigated: PS colloidal crystals, PS colloidal crystals covered by $\mathrm{Al}$, and silica crystals, with different sphere diameters of $420 \pm 9 \mathrm{~nm}, 376 \pm 8 \mathrm{~nm}$ and $238 \pm 7 \mathrm{~nm}$, respectively. Due to the growing method, the dried colloidal crystal films used in our experiment contain cracks 
between single-crystal regions of about $50 \mu \mathrm{m}$ in size. Microscopic images of these samples are shown in Figure 2.

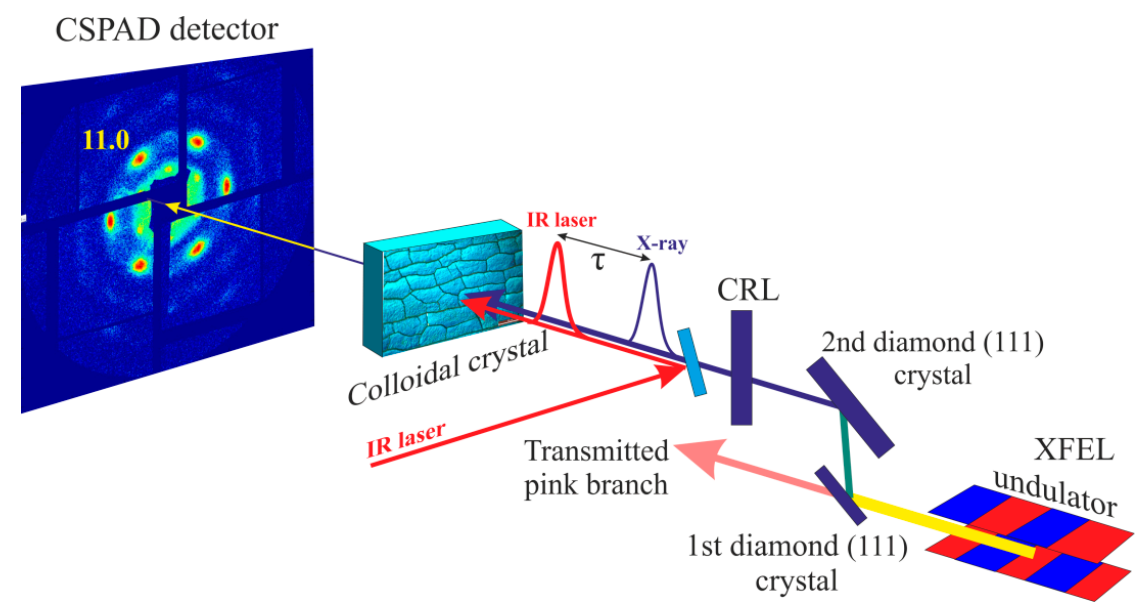

Figure 1. The scheme of the pump-probe experiment. X-ray free-electron laser (XFEL) pulses are generated by the undulator and reflected by the first diamond crystal. The second diamond crystal reflects the beam in the direction to the sample position. The beam is focused by the compound refractive lenses (CRLs) to the size of $50 \mu \mathrm{m}$ at the sample position. The Cornell-SLAC Pixel Array Detector (CSPAD) is positioned $10 \mathrm{~m}$ downstream from the colloidal sample.
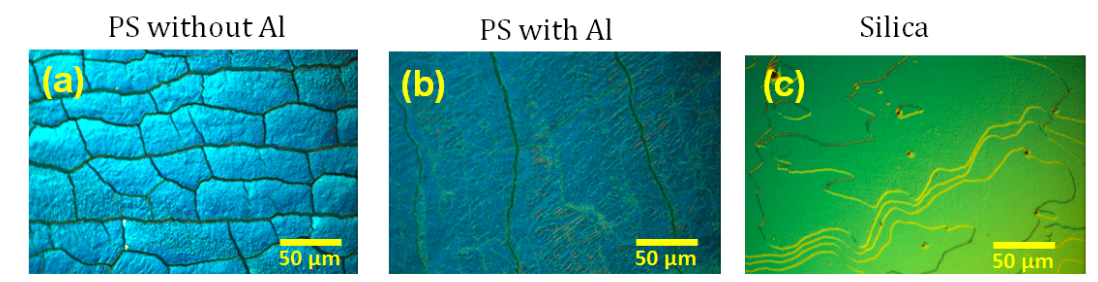

Figure 2. Microscopic images of colloidal crystals made from (a) polystyrene (PS); (b) PS covered with an Al layer; and (c) silica samples measured at Linac Coherent Light Source (LCLS).

Series of X-ray diffraction images were recorded using the Cornell-SLAC Pixel Array Detector (CSPAD) megapixel X-ray detector [24], positioned $10 \mathrm{~m}$ downstream from the sample, that is comprised of 32 silicon sensors with the pixel size of $110 \times 110 \mu^{2}$ covering an area approximately $17 \times 17 \mathrm{~cm}^{2}$. This experimental arrangement provided resolution of $0.5 \mu \mathrm{m}^{-1}$ per pixel in reciprocal space. The 2.3 megapixels of the detector were read out at $120 \mathrm{~Hz}$, encoded at 14 bits per pixel. In order to prevent damage of the detector, a $5 \times 5 \mathrm{~mm}^{2} \mathrm{Al}$ beamstop was placed in front of it. To reduce air scattering, a $10 \mathrm{~m}$ long flight tube filled with He was mounted between the sample and the detector.

A Ti:sapphire IR laser was used to excite the colloidal crystal film. The pump pulses were generated with a wavelength $\lambda=800 \mathrm{~nm}(1.5497 \mathrm{eV})$ and duration about $50 \mathrm{fs}$ (FWHM). The size of the laser footprint on the sample was $100 \mu \mathrm{m}$ (FWHM) and, thus, twice larger in comparison to the X-ray beam. The corresponding intensity of the laser pulses was on the order of $10^{13} \mathrm{~W} / \mathrm{cm}^{2}$ " The IR laser pulses were propagating along the XFEL pulses and were synchronized to the pulses from the XFEL with less than $0.5 \mathrm{ps}$ jitter.

The IR laser energy was controlled by rotation of the optical axis of a waveplate (see Figure 1), and was calibrated by power sensor at the position of the sample. The measured calibration curve is presented in Figure 3. Zero degrees of waveplate angle corresponds to the minimum and 10 degrees correspond to the maximum calibrated energy and intensity of the IR laser.

A series of pump-probe experiments were performed with the variation of the IR laser energy from $195 \mu \mathrm{J}$ to $691 \mu \mathrm{J}\left(3.2 \times 10^{13}-1.1 \times 10^{14} \mathrm{~W} / \mathrm{cm}^{2}\right)$ for PS and silica samples and from $195 \mu \mathrm{J}$ to 
$326 \mu \mathrm{J}\left(3.2 \times 10^{13}-5.4 \times 10^{13} \mathrm{~W} / \mathrm{cm}^{2}\right)$ for PS films covered by Al. Measurements with different IR laser energies were performed at the new position of the sample to avoid sample damage. The upper limit of IR laser energies was set due to the damage threshold of the colloidal crystals and was lower for Al-covered samples due to enhanced absorption of IR light by the Al film. The results for the highest non-destructive IR laser energies, which are marked with arrows on the calibration curve, are presented in this work. At energies higher than $1 \mathrm{~mJ}$, ultrafast melting of the colloidal crystals was observed and was investigated in a separate work [25].

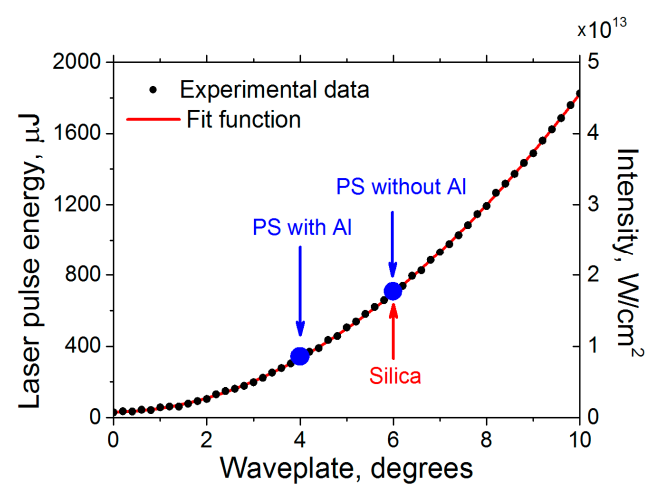

Figure 3. The infrared (IR) laser energy calibration curve. The maximum of the IR laser energy used in the experiments for three samples is marked with arrows. For the PS colloidal crystal covered with Al layer, the maximum IR laser energy was $326 \mu \mathrm{J}$, and for the pure PS and silica samples, $691 \mu \mathrm{J}$.

The pump-probe experiment was performed with a time delay variation $\tau$ from -50 ps to $+1000 \mathrm{ps}$, with a $10 \mathrm{ps}$ time increment. This time delay region and the time interval were considered in order to resolve $\mathrm{GHz}$ frequency dynamics of colloidal crystals. In order to obtain sufficient statistics of the measured data, for each time delay a large number of diffraction patterns (600 for polystyrene and 120 for silica) with and without the IR laser were measured. Typical single-shot diffraction patterns for three different samples are shown in Figure 4 . The six-fold symmetry of the diffraction pattern is due to the hexagonal close-packed structure of the colloidal crystals. Two orders of Bragg peaks can be seen in single diffraction patterns shown in Figure 4. A family of 11.0 Bragg reflections, indicated in Figure 4 by squares, was used in further analysis. Indices of the structure are adopted from the hexagonal structure and the point symbolizes the fourth index equal to the negative sum of the first two indices (11.0 equals to $11 \overline{2} 0$ ).

PS without Al

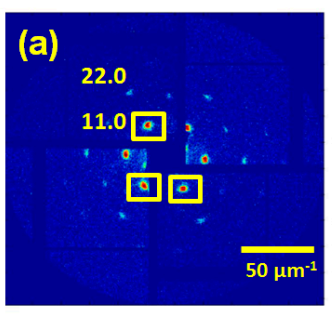

PS with Al

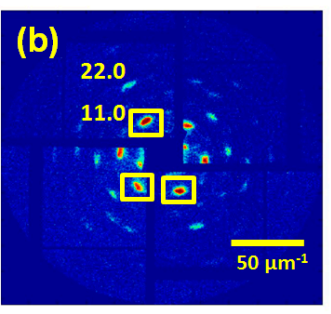

Silica

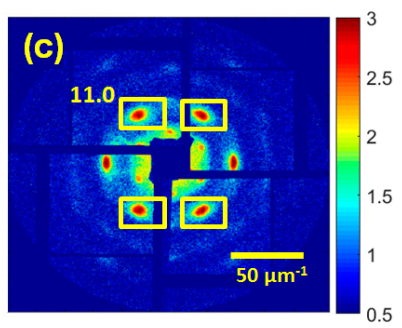

Figure 4. Single-shot diffraction patterns (in logarithmic scale) from colloidal crystals made from (a) PS; (b) PS covered with Al layer, and (c) silica samples measured at LCLS. Squares indicate Bragg peaks considered in the further analysis.

\section{Data Analysis}

The following strategy was implemented for the data analysis. First, for the patterns with sufficiently high intensity (more than 5000 counts per single diffraction pattern) of the XFEL pulse, 
we selected Bragg peaks located far away from the detector gaps (see Figure 4). Patterns with a lower signal were not treated. Using this procedure, typically three to four Bragg peaks were selected for the analysis (see Figure 4). Next, the Bragg peak positions were extracted using the center of mass of their intensity distributions as well as the relative distances between the opposite Bragg peaks. The change of the relative positions of the Bragg peaks as a function of the time delay allowed us to probe the lattice dynamics along specific crystal directions. The following parameters as a function of time delay were analyzed in order to reveal the dynamics of the colloidal crystals, induced by the IR laser: Bragg peak position $q(\tau)$, as well as FWHMs in the radial $w_{q}(\tau)$ and azimuthal $w_{\phi}(\tau)$ directions in reciprocal space. The temporal variation of the momentum transfer vector $q(\tau)$ is related to the dynamics in the inter-particle spacing, while $w_{q}(\tau)$ corresponds to the dynamics of the average particle size, and $w_{\phi}(\tau)$ defines angular misorientation of coherent scattering domains [26].

Due to the nature of the SASE process, each XFEL pulse has a unique fine spatial structure that was mapped by the intensity distribution at each Bragg position. A multi-spiked structure of the Bragg peaks varying from pulse to pulse was observed in our experiment (see Figure 5). From these diffraction patterns spatial and temporal coherence properties of hard XFEL could be determined by spatial correlation analysis [27]. Due to the different shape of each FEL pulse (see Figure 5), it was not possible to perform deconvolution of each scattered pulse shape. For the same reason (non-Gaussian structure of Bragg peaks) fitting of the peaks with the two-dimensional Gaussian function was not reliable (see Figure 6a). In order to improve Bragg peak characterization, projections of their intensities on azimuthal and radial directions were performed. These data were fitted with the one-dimensional Gaussian function (see Figure $6 \mathrm{~b})$, and peak broadening in the radial $\left(w_{q}\right)$ and azimuthal $\left(w_{\phi}\right)$ directions was determined.
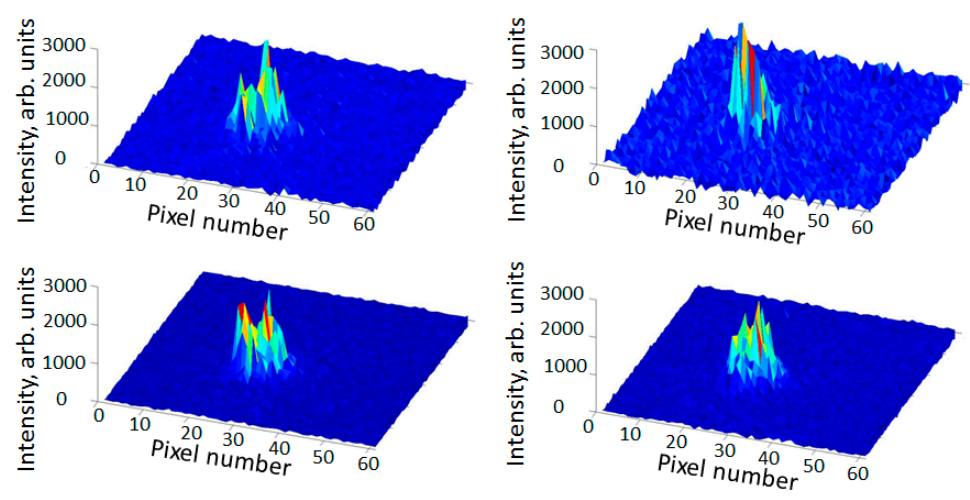

Figure 5. Four single-shot diffraction patterns measured at the same Bragg peak position. The spike-shaped peaks demonstrate an influence of the individual structure of each XFEL pulse on the Bragg peak.
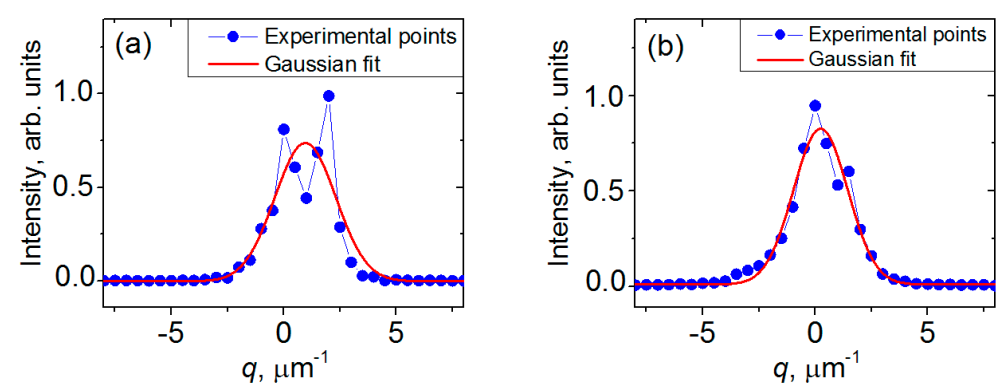

Figure 6. (a) The cross-section of a two-dimensional Bragg peak along azimuthal direction and (b) the projection of the same Bragg peak on the same direction . It is seen that the cross-section dataset (a) deviates from the Gaussian fit (red line), whereas the projection points are fitted with the Gaussian function with higher accuracy. 
In order to compare dynamics of the collected data as a function of the time delay $\tau$ for different samples and IR laser energies, the following dimensionless parameters were used:

$$
\begin{aligned}
\frac{\Delta q(\tau)}{q} & =\frac{\left\langle q_{o n}(\tau)\right\rangle-\left\langle q_{o f f}\right\rangle}{\left\langle q_{o f f}\right\rangle} \\
\frac{\Delta w(\tau)}{w} & =\frac{\left\langle w_{o n}(\tau)\right\rangle-\left\langle w_{o f f}\right\rangle}{\left\langle w_{o f f}\right\rangle}
\end{aligned}
$$

where $q(\tau)$ is the distance between the opposite Bragg peaks, and $w(\tau)$ is the radial or azimuthal FWHM of the Bragg peak. Subscripts "on" and "off" define measurements with and without the IR laser, respectively. Brackets $\langle\ldots\rangle$ denote ensemble averaging of the chosen Bragg peak parameter for each time delay $\tau$ over all XFEL pulses. Time dependencies of the momentum transfer vector $q(\tau)$, as well as radial $w_{q}(\tau)$ and azimuthal $w_{\phi}(\tau)$ broadening of the Bragg peaks for three measured samples, are shown in Figure 7. Some points in this figure are excluded due to the unexpected drops of intensity of the XFEL (such drops of intensity were observed when operation of some clystrons in the accelerator complex was failing). The statistical error of these parameters was determined using the standard approach for the error determination in the multi-parameter equation [28]. As clearly seen in Figure 7, an error of the $\Delta q(\tau) / q$ values is one order of magnitude lower than for the Bragg peaks broadening parameters. This could be explained by the influence of the XFEL pulse shape on the shape of the Bragg peaks with a relatively stable position of each pulse. Due to this, the Gaussian fit of the Bragg peaks shape was not accurate, while the influence of the XFEL pulse shape on the variation of the center of mass was insignificant. The error values for the silica sample are higher than for the PS due to lower statistics (for the PS crystals 600 shots and for the silica crystals 120 shots were collected with the IR laser).
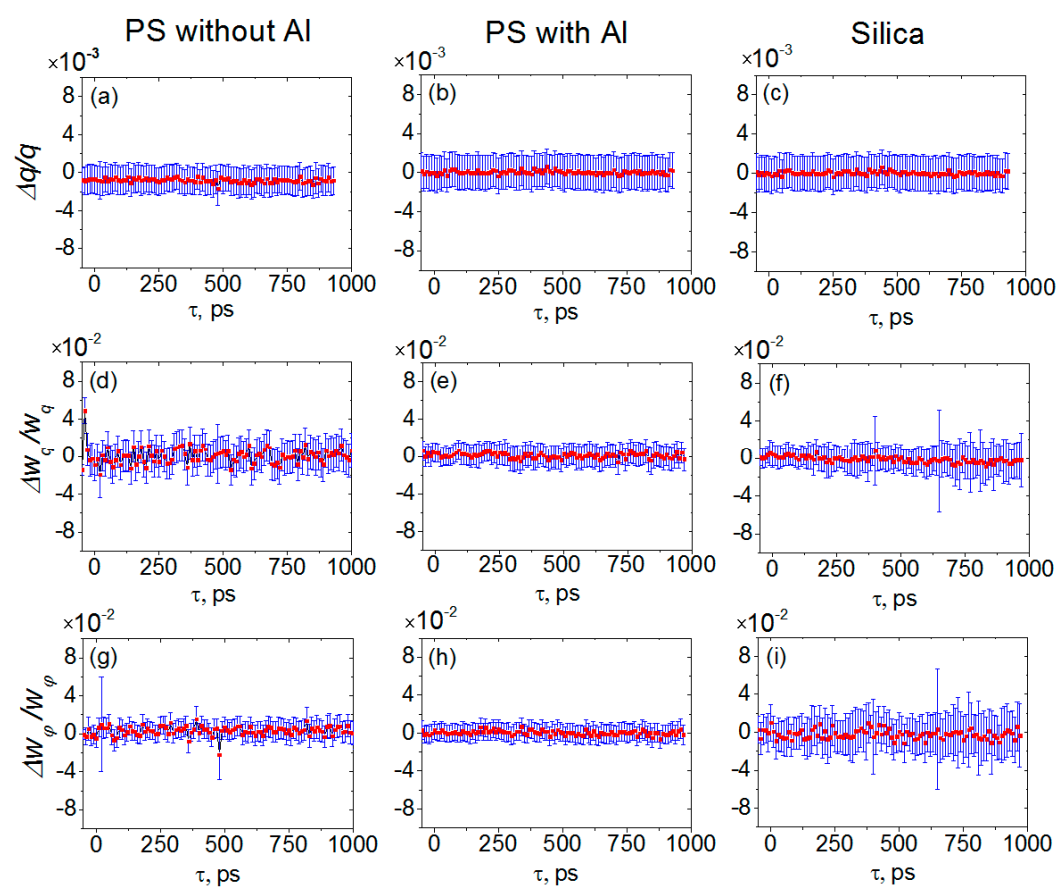

Figure 7. Time dependence of the relative change of the distance between the two opposite Bragg peaks $\Delta q(\tau) / q(\mathbf{a}-\mathbf{c})$ and Bragg peaks widths in radial $\Delta w_{q}(\tau) / w_{q}(\mathbf{d}-\mathbf{f})$ and azimuthal $\Delta w_{\phi}(\tau) / w_{\phi}$ $(\mathrm{g}-\mathbf{i})$ directions for three selected samples. Analysis of the data was performed for the IR pump laser intensities shown in Figure 3 and 11.0 Bragg peaks indicated by a square in Figure 4. 
Figure 7 also clearly shows that variations of all parameters are around zero and in all cases, the signal is lower than the statistical error. To reveal characteristic frequencies excited by the IR laser, we performed the Fourier analysis of the time dependencies of the Bragg peak parameters described above. The corresponding Fourier spectra are shown in Figure 8. Due to the more precise measurements of the momentum transfer vector $q(\tau)$, the average value of the Fourier components of $q(\tau)$ is one order of magnitude lower than for the Bragg peaks broadening $w(\tau)$. There was no significant enhancement of any particular Fourier component, for all Fourier spectra. We determined an average value of the Fourier components and the standard deviation $\sigma$ from the distribution of these Fourier components. For all Fourier spectra shown in Figure 8, there is no Fourier component higher than $3 \sigma$ above the average Fourier component value, which indicates that the periodic signal could not be reliably detected in these conditions.
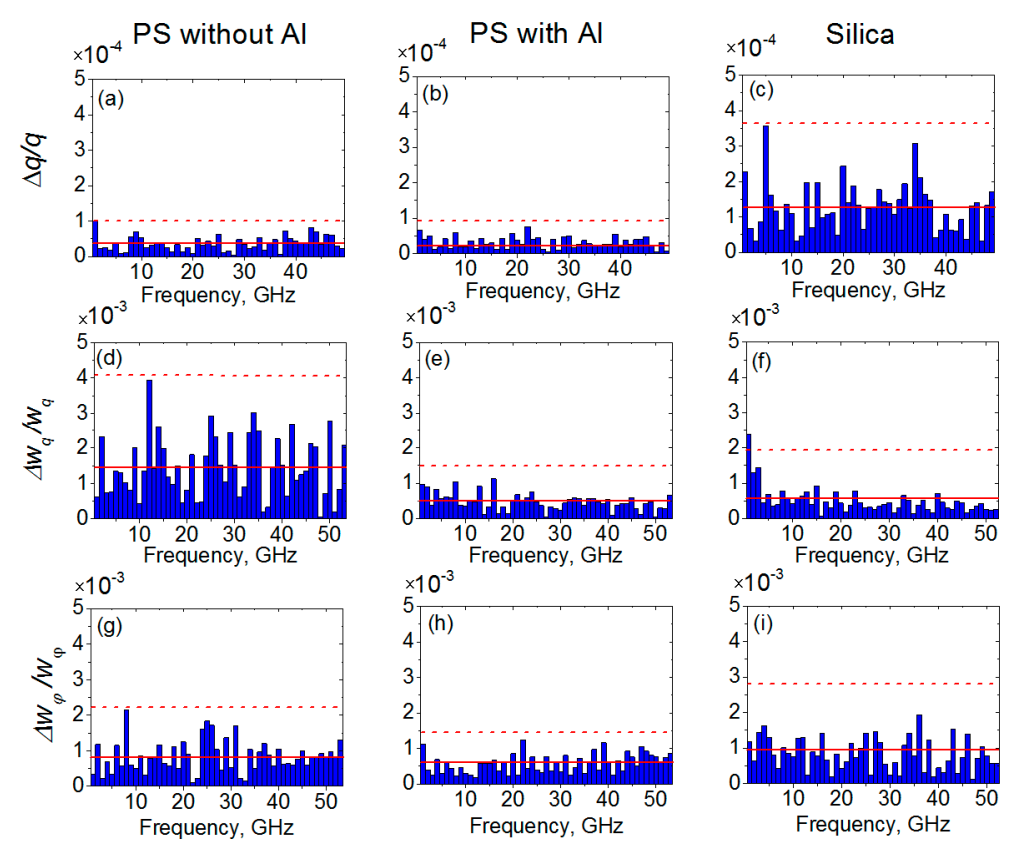

Figure 8. Fourier spectra from the distance between the two opposite Bragg peaks $\Delta q(\tau) / q(\mathbf{a}-\mathbf{c})$ and Bragg peaks widths in radial $\Delta w_{q}(\tau) / w_{q}(\mathbf{d}-\mathbf{f})$ and azimuthal $\Delta w_{\phi}(\tau) / w_{\phi}(\mathbf{g}-\mathbf{i})$ directions for three selected samples. Fourier spectra calculated from the results of diffraction pattern analysis shown in Figure 7. Average value of all Fourier components is indicated by the red line. The dashed red line corresponds to the $3 \sigma$ level above the average value, where $\sigma$ is the standard deviation and is determined from the distribution of Fourier components.

\section{Model Simulations}

To have better understanding of the obtained results, we performed simulations of the colloidal crystal dynamics induced by an external IR pulse excitation. In our model colloidal crystals consist of isotropic homogeneous spheres. The characteristic frequencies of vibration of a colloidal particle were determined using the Lamb theory describing vibrations of an isotropic elastic sphere [29]. Theoretically, two families of Lamb modes, namely spheroidal and torsional ones, can be derived from the equations of motion. The spheroidal modes cause the change of the sphere volume, and the torsional modes leave the sphere volume unperturbed. During the IR pulse propagation through a sphere, the changes of the sphere volume are expected and, therefore, we used spheroidal modes in our simulations. We determined the frequencies of the first spheroidal mode for all three measured samples using reference [30]. Sphere diameter, longitudinal $c_{L}$ and transverse $c_{T}$ sound velocities of PS and silica were obtained from reference [31]. These parameters, as well as the frequencies of the first spheroidal mode for the colloidal crystals, are summarized in Table 1. 
Table 1. Parameters of PS and silica nanoparticles used in our model. The corresponding frequencies of the first spheroidal mode of colloidal particles were obtained from reference [30]. The noise level was derived from the experimental data. Relative and absolute deviation in the interparticle distance $\left(\Delta d / d_{0}\right.$ and $\left.\Delta d\right)$, which could be detected in our experiment with the probability of $60 \%$, were calculated from the experimental data and simulations.

\begin{tabular}{cccc}
\hline & PS without Al & PS with Al & Silica \\
\hline Sphere diameter, $\mathrm{nm}$ & 420 & 376 & 238 \\
Longitudinal sound velocity $c_{L}, \mathrm{~m} / \mathrm{s}$ & 2350 & 2350 & 5950 \\
Transverse sound velocity $c_{T}, \mathrm{~m} / \mathrm{s}$ & 1210 & 1210 & 3760 \\
Frequency of the breathing mode, $\mathrm{GHz}$ & 4.97 & 5.54 & 19.06 \\
Noise level $N \times 10^{-3}$ & 1.4 & 1.8 & 1.8 \\
Relative deviation in interparticle distance $\Delta d / d_{0} \times 10^{-3}$ & 0.7 & 0.9 & 0.9 \\
Deviation in interparticle distance $\Delta d, \mathrm{~nm}$ & 0.29 & 0.35 & 0.22 \\
\hline
\end{tabular}

The values of $\Delta q(\tau) / q$ were obtained from the experimental data with the smallest error in comparison to other Bragg peak parameters for all investigated samples (see Figure 7a-c). Therefore, the parameter $\Delta q(\tau) / q$ was considered for our simulations. Assuming an ideal close-packed crystal with the interparticle distances equal to the particles diameter, the unperturbed momentum transfer modulus of the 11.0 Bragg reflection can be described as $q_{0}=2 \pi / d_{11.0}=4 \pi / d_{0}$, where $d_{0}$ is the unperturbed particle diameter. We simulated vibrations of the colloidal spheres with the diameter $d_{0}$ as a periodic sinusoidal signal:

$$
d(\tau)=d_{0}(1-S \cdot \sin (\omega \tau))
$$

where $S$ is the relative amplitude and $\omega$ is the characteristic frequency of the vibration. The dynamics of the experimentally-observed 11.0 diffraction peak can be described as:

$$
q(\tau)=\frac{4 \pi}{d(\tau)} \approx \frac{4 \pi}{d_{0}}(1+S \cdot \sin (\omega \tau))
$$

where we used the Taylor series expansion of the momentum transfer vector $q(\tau)$ due to the small values of the amplitude $S<1$.

As a result, measured changes in the Bragg peak positions and $\Delta q(\tau) / q$ can be simulated as a periodic sinusoidal signal. We should also take into account systematic errors of the measurement as an additional noise. Finally, simulations of the parameter $\Delta q(\tau) / q$ in our model were described using the following expression:

$$
\frac{\Delta q(\tau)}{q_{0}}=\frac{q(\tau)-q_{0}}{q_{0}}=S \cdot \sin (\omega \tau)+N \cdot \eta_{\text {noise }}(\tau)
$$

where $N$ is the amplitude of a noise and $\eta_{\text {noise }}(\tau)$ is a random noise function with normal distribution and standard deviation value equal to one. The signal-to-noise ratio in this case is assumed to be equal to the ratio of the amplitudes $S / N$.

Next, we defined criterion when the periodic signal is detected by the algorithm in our simulations. Due to the random behavior of the noise function $\eta_{\text {noise }}(\tau)$, each realization of the signal $\Delta q(\tau) / q_{0}$ (see Equation (5)) for a constant $S / N$ is different. As an example, we demonstrate this for the experimental data of the polystyrene sample without aluminum. This sample was considered as one with the lowest noise level in our experiment. The time interval and increment of the simulated signal $\Delta q(\tau) / q_{0}$ were chosen the same as in our pump-probe experiment. One of the typical realizations of the $\Delta q(\tau) / q_{0}$ with the signal level $S=0.7 \times 10^{-3}$ and noise level $N=1.4 \times 10^{-3}$ is shown in Figure 9a. From this figure it is difficult to conclude on the presence or absence of the signal. 

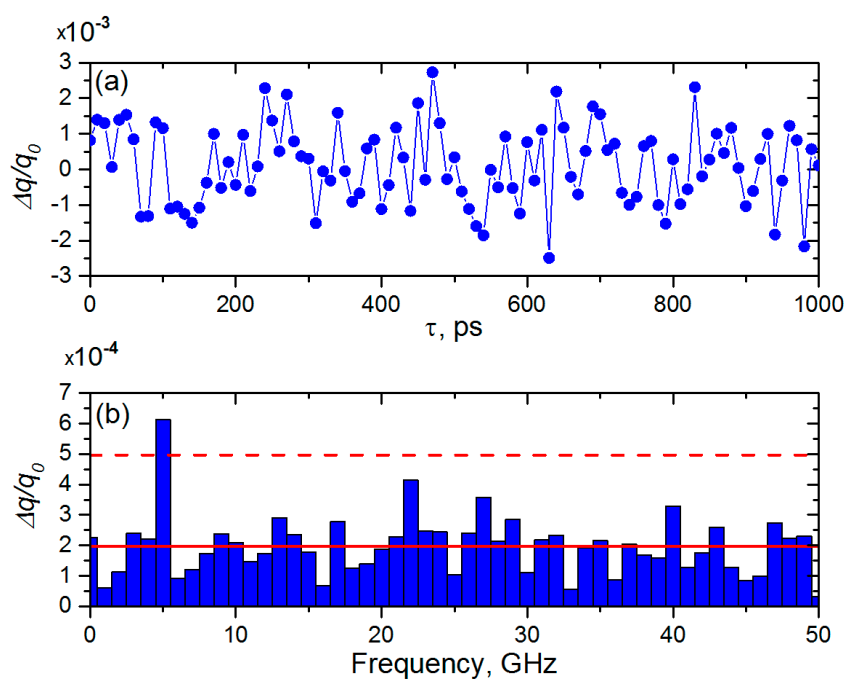

Figure 9. (a) Simulation of the signal $\Delta q(\tau) / q_{0}$ and (b) Fourier transform of this signal. The average value of all Fourier components is indicated by red line. The dashed red line corresponds to level of $3 \sigma$ above the average value, where $\sigma$ is a standard deviation and is determined from the distribution of Fourier components.

Similar to the analysis of the experimental data, the Fourier spectra were calculated for the simulated signal with the noise (see Figure $9 b$ ). Next, the behavior of the Fourier component amplitude $\left(A_{\omega}\right)$ corresponding to the first spheroidal mode frequency $\omega=4.97 \mathrm{GHz}$ was studied. The criterion of detecting the signal was considered as:

$$
A_{\omega}>\bar{A}+3 \sigma
$$

where $\bar{A}$ is the mean and $\sigma$ is the standard deviation of all Fourier components in the spectrum. The Fourier spectrum of the simulated data (see Figure 9a), as well as its $3 \sigma$ level are shown in Figure $9 \mathrm{~b}$. It can be seen that the contribution of the vibrational mode in the Fourier spectrum is higher than the criterion stipulated by Equation (6). Therefore, the algorithm detected the presence of the $4.97 \mathrm{GHz}$ frequency signal.

We calculated the probability of successful signal detection as a function of signal-to-noise ratio $S / N$ in the range of $(S / N)$ from 0 to 1.5 with 0.01 increment. During the simulations the amplitude of the signal $S$ was changing, while the noise level $N$ was kept constant. The probability of detecting signal $P(S / N)$ was defined as:

$$
P(S / N)=\lim _{n \rightarrow \infty}\left(\frac{n_{\text {detected }}(S / N)}{n}\right)
$$

where $n_{\text {detected }}(S / N)$ is a number of simulations with successful detection of signal (according to the criterion stipulated by Equation (6)) and $n=10,000$ is the total number of simulations for each $S / N$ step. Results of these simulations for the experimental data of the polystyrene sample without aluminum are shown in Figure 10. As one can see from Figure 10 that, according to our criterion stipulated by Equation (6), the signal is $100 \%$ detectable if it is on the level of the noise $(S / N=1)$. 


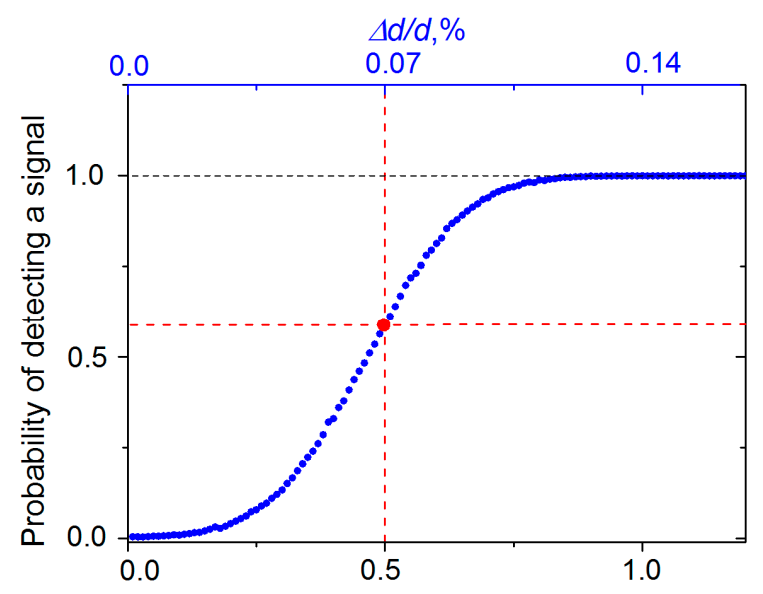

Figure 10. The probability of successful signal detection as a function of signal-to-noise ratio $(S / N)$. On the top horizontal axis, the change of the corresponding interparticle distance for the polystyrene sample without $\mathrm{Al}$ is shown. The red lines correspond to the value of $S / N=0.5$ and $P(S / N)=0.6$.

\section{Results and Discussion}

Simulations, described in the previous section, were performed for each sample (polystyrene sample without aluminum, polystyrene sample with aluminum, and silica samples) to derive the probability function $P(S / N)$ of detecting the periodic signal (see Figure 10) in each case. It is clearly visible from Figure 10 that even the value of the signal-to-noise ratio $S / N=0.5$ gives $60 \%$ probability of detecting the signal. This condition was considered as a criterion for the minimum measurable signal. Using well-known relations between the deviations of the momentum transfer vectors and interparticle distances $\Delta q(\tau) / q_{0}=-\Delta d(\tau) / d_{0}$, as well as Equation (5), we can determine the relative (and absolute) values of the changes of the interparticle distances $\Delta d(\tau) / d_{0}(\Delta d(\tau))$ for the given values of the experimental noise $N$. As a result of this analysis, we can obtain the minimum values of these parameters which could be detected in our experimental conditions with the probability of $60 \%$. For all measured samples results of this analysis are summarized in Table 1.

As it follows from Table 1, the minimum relative changes of the interparticle distance $\Delta d / d_{0}$ that can be detected with the probability of $60 \%$ for all samples were about $10^{-3}$. Such values correspond to relatively large amplitudes of the deviations in interparticle distances of about $0.2-0.4 \mathrm{~nm}$. Clearly, in our experimental conditions excited vibrations were lower than the noise level of the experiment that prevented us from their experimental determination.

For the successful measurement of vibrations of colloidal crystals at the FEL sources, some further improvements of the experiment may be suggested. First, the accuracy of the measurement of the incoming X-ray beam intensity from pulse to pulse should be improved, as this would allow measurement of the Bragg peak intensity with higher precision. In our experiment such an accurate measurement of the Bragg peak intensity variation was not possible due to the complicated structure of each XFEL pulse and a relatively low accuracy (about 3\%) in the measurement of the incoming X-ray beam intensity from pulse to pulse. Second, colloidal crystals may be covered by a thicker aluminum layer which could lead to a more efficient energy transfer from an IR laser to the colloidal crystal [32]. This would lead to a higher amplitude of vibrations of a PS colloidal crystal, and enable their detection in IR pump and X-ray probe experiments.

\section{Conclusions}

We performed experiments at the LCLS to investigate dynamics in the colloidal crystals. The diffraction patterns from the colloidal crystals, which were pumped by IR laser pulses and probed by XFEL radiation, were collected. The measurements were performed in a non-destructive regime at the different positions of the samples. The pump-probe experiments were performed in the 
wide range of the IR laser energies, from $195 \mu \mathrm{J}$ up to the upper limit of the non-destructive threshold of $691 \mu \mathrm{J}$.

Through the analysis of the Bragg peaks, extracted from the diffraction patterns, changes in the colloidal crystals caused by the IR laser were investigated. The dynamics at different timescales was studied by the Fourier analysis of parameters associated with the momentum transfer and Bragg peak broadening in the radial and angular directions. Simulations of the colloidal crystal dynamics were performed and the probability function $P(S / N)$ was derived. Relative and absolute deviations in the interparticle distance $\left(\Delta d / d_{0}\right.$ and $\left.\Delta d\right)$, which could be detected in such an experiment with the probability of $60 \%$ (corresponding to the signal to noise level of 0.5 ), were calculated for all measured samples. From the experiment and simulations, we were able to conclude that, for all measured samples, the amplitudes of vibrational modes excited in colloidal crystals were less than the systematic error caused by the noise level.

In future experiments with XFEL sources different scattering geometries may be foreseen. For example, performing scattering experiments on colloidal crystals in grazing incidence small-angle X-ray scattering (GISAXS) [33,34] may increase the sensitivity of the scattered radiation to changes induced by the IR pump laser.

Acknowledgments: We acknowledge fruitful discussions and support of the project by Edgar Weckert as well as help and support by Andrei V. Petukhov. We acknowledge the help of Ralf Röhlsberger in preparation of Al-covered colloidal crystals. We acknowledge careful reading of the manuscript by Tim Laarman. This work was supported by the Virtual Institute VH-VI-403 of the Helmholtz Association. The experimental work was carried out at the Linac Coherent Light Source (LCLS), a National User Facility operated by Stanford University on behalf of the U.S. Department of Energy, Office of Basic Energy Sciences. Use of the LCLS, SLAC National Accelerator Laboratory, is supported by the U.S. Department of Energy, Office of Science, and Office of Basic Energy Sciences under contract number DE-AC02-76SF00515. A.S. was supported by U.S. Department of Energy, Office of Science, Office of Basic Energy Sciences, under Contracts No. DE-SC0001805. For G.W. portions of this research were carried out at Brookhaven National Laboratory, operated under Contract No. DE-SC0012704 from the U.S. Department of Energy (DOE) Office of Science.

Author Contributions: M.C., A.S., R.P.K., O.M.Y., D.D., O.Y.G., G.W., D.Z., Y.F., M.S., S.S., and I.A.V. performed the experiments; J.-M.M. and T.G. prepared the colloidal crystal samples; I.A.V. supervised N.M. and the project; N.M., A.G.S., E.A.S., and S.L. developed the code; N.M. analyzed the data; and N.M. and S.L. wrote the paper.

Conflicts of Interest: The authors declare no conflict of interest.

\section{References}

1. Gorishnyy, T.; Ullal, C.K.; Maldovan, M.; Fytas, G.; Thomas, E. Hypersonic phononic crystals. Phys. Rev. Lett. 2005, 94, 115501. [CrossRef] [PubMed]

2. Cheng, W.; Wang, J.; Jonas, U.; Fytas, G.; Stefanou, N. Observation and tuning of hypersonic bandgaps in colloidal crystals. Nat. Mater. 2006, 5, 830. [CrossRef] [PubMed]

3. Still, T.; Cheng, W.; Retsch, M.; Sainidou, R.; Wang, J.; Jonas, U.; Stefanou, N.; Fytas, G. Simultaneous occurrence of structure-directed and particle-resonance-induced phononic gaps in colloidal films. Phys. Rev. Lett. 2008, 100, 194301. [CrossRef] [PubMed]

4. Thomas, E.L.; Gorishnyy, T.; Maldovan, M. Phononics: Colloidal crystals go hypersonic. Nat. Mater. 2006, 5, 773-774. [CrossRef] [PubMed]

5. Akimov, A.; Tanaka, Y.; Pevtsov, A.; Kaplan, S.; Golubev, V.; Tamura, S.; Yakovlev, D.; Bayer, M. Hypersonic modulation of light in three-dimensional photonic and phononic band-gap materials. Phys. Rev. Lett. 2008, 101, 033902. [CrossRef] [PubMed]

6. Salasyuk, A.S.; Scherbakov, A.V.; Yakovlev, D.R.; Akimov, A.V.; Kaplyanskii, A.A.; Kaplan, S.F.; Grudinkin, S.A.; Nashchekin, A.V.; Pevtsov, A.B.; Golubev, V.G.; et al. Filtering of elastic waves by opal-based hypersonic crystal. Nano Lett. 2010, 10, 1319-1323. [CrossRef] [PubMed]

7. Mazurenko, D.; Shan, X.; Stiefelhagen, J.; Graf, C.; Van Blaaderen, A.; Dijkhuis, J. Coherent vibrations of submicron spherical gold shells in a photonic crystal. Phys. Rev. B 2007, 75, 161102. [CrossRef]

8. Duval, E.; Boukenter, A.; Champagnon, B. Vibration eigenmodes and size of microcrystallites in glass: Observation by very-low-frequency Raman scattering. Phys. Rev. Lett. 1986, 56, 2052. [CrossRef] [PubMed] 
9. Emma, P.; Akre, R.; Arthur, J.; Bionta, R.; Bostedt, C.; Bozek, J.; Brachmann, A.; Bucksbaum, P.; Coffee, R.; Decker, F.J.; et al. First lasing and operation of an ångstrom-wavelength free-electron laser. Nat. Photonics 2010, 4, 641-647. [CrossRef]

10. Altarelli, M.; Brinkmann, R.; Chergui, M.; Decking, W.; Dobson, B.; Dusterer, S.; Grubel, G.; Graeff, W.; Graafsma, H.; Hajdu, J.; et al. XFEL The European X-ray Free-Electron Laser; Technical Design Report, DESY Report No. 2006-097; DESY XFEL Project Group, European XFEL Project Team, Deutsches Elektronen-Synchrotron, Member of the Helmholtz Association: Hamburg, Germany, 2006; Available online: http:/ / xfel.desy.de/technical_information/tdr/tdr/ (accessed on 17 May 2017).

11. Ishikawa, T.; Aoyagi, H.; Asaka, T.; Asano, Y.; Azumi, N.; Bizen, T.; Ego, H.; Fukami, K.; Fukui, T.; Furukawa, Y.; et al. A compact X-ray free-electron laser emitting in the sub-ångstrom region. Nat. Photonics 2012, 6, 540-544. [CrossRef]

12. McFarland, B.; Farrell, J.; Miyabe, S.; Tarantelli, F.; Aguilar, A.; Berrah, N.; Bostedt, C.; Bozek, J.; Bucksbaum, P.; Castagna, J.; et al. Ultrafast X-ray Auger probing of photoexcited molecular dynamics. Nat. Commun. 2014, 5. [CrossRef] [PubMed]

13. Trigo, M.; Fuchs, M.; Chen, J.; Jiang, M.; Cammarata, M.; Fahy, S.; Fritz, D.; Gaffney, K.; Ghimire, S.; Higginbotham, A.; et al. Fourier-transform inelastic X-ray scattering from time-and momentum-dependent phonon-phonon correlations. Nat. Phys. 2013, 9, 790-794. [CrossRef]

14. Kupitz, C.; Basu, S.; Grotjohann, I.; Fromme, R.; Zatsepin, N.A.; Rendek, K.N.; Hunter, M.S.; Shoeman, R.L.; White, T.A.; Wang, D.; et al. Serial time-resolved crystallography of photosystem II using a femtosecond X-ray laser. Nature 2014, 513, 261-265. [CrossRef] [PubMed]

15. Glownia, J.; Natan, A.; Cryan, J.; Hartsock, R.; Kozina, M.; Minitti, M.; Nelson, S.; Robinson, J.; Sato, T.; van Driel, T.; et al. Self-referenced coherent diffraction X-ray movie of Ångstrom-and femtosecond-scale atomic motion. Phys. Rev. Lett. 2016, 117, 153003. [CrossRef] [PubMed]

16. Abbey, B.; Dilanian, R.A.; Darmanin, C.; Ryan, R.A.; Putkunz, C.T.; Martin, A.V.; Wood, D.; Streltsov, V.; Jones, M.W.; Gaffney, N.; et al. X-ray laser-induced electron dynamics observed by femtosecond diffraction from nanocrystals of Buckminsterfullerene. Sci. Adv. 2016, 2, e1601186. [CrossRef] [PubMed]

17. Clark, J.; Beitra, L.; Xiong, G.; Higginbotham, A.; Fritz, D.; Lemke, H.; Zhu, D.; Chollet, M.; Williams, G.; Messerschmidt, M.; et al. Ultrafast three-dimensional imaging of lattice dynamics in individual gold nanocrystals. Science 2013, 341, 56-59. [CrossRef] [PubMed]

18. Dronyak, R.; Gulden, J.; Yefanov, O.; Singer, A.; Gorniak, T.; Senkbeil, T.; Meijer, J.M.; Al-Shemmary, A.; Hallmann, J.; Mai, D.; et al. Dynamics of colloidal crystals studied by pump-probe experiments at FLASH. Phys. Rev. B 2012, 86, 064303. [CrossRef]

19. Chollet, M.; Alonso-Mori, R.; Cammarata, M.; Damiani, D.; Defever, J.; Delor, J.T.; Feng, Y.; Glownia, J.M.; Langton, J.B.; Nelson, S.; et al. The X-ray pump-probe instrument at the Linac Coherent Light Source. J. Synchrotron Radiat. 2015, 22, 503-507. [CrossRef] [PubMed]

20. Saldin, E.L.; Schneidmiller, E.A.; Yurkov, M.V. The Physics of Free Electron Lasers; Springer: Berlin, Germany, 2000.

21. Stoupin, S.; Terentyev, S.; Blank, V.; Shvyd'ko, Y.V.; Goetze, K.; Assoufid, L.; Polyakov, S.; Kuznetsov, M.; Kornilov, N.; Katsoudas, J.; et al. All-diamond optical assemblies for a beam-multiplexing X-ray monochromator at the Linac Coherent Light Source. J. Appl. Crystallogr. 2014, 47, 1329-1336. [CrossRef] [PubMed]

22. Zhu, D.; Feng, Y.; Stoupin, S.; Terentyev, S.A.; Lemke, H.T.; Fritz, D.M.; Chollet, M.; Glownia, J.; Alonso-Mori, R.; Sikorski, M.; et al. Performance of a beam-multiplexing diamond crystal monochromator at the Linac Coherent Light Source. Rev. Sci. Instrum. 2014, 85, 063106. [CrossRef] [PubMed]

23. Meijer, J.-M. Colloidal Crystals of Spheres and Cubes in Real and Reciprocal Space; Springer: Berlin, Germany, 2015; pp. 23-29.

24. Hart, P.; Boutet, S.; Carini, G.; Dubrovin, M.; Duda, B.; Fritz, D.; Haller, G.; Herbst, R.; Herrmann, S.; Kenney, C.; et al. The CSPAD megapixel X-ray camera at LCLS. In Proc. SPIE 8504, X-ray Free-Electron Lasers: Beam Diagnostics, Beamline Instrumentation, and Applications, 85040C; Moeller, S.P., Yabashi, M., Hau-Riege, S.P., Eds.; SPIE: Bellingham, WA, USA, 2012.

25. Lazarev, S.; Mukharamova, N.; Gorobtsov, O.Y.; Chollet, M.; Kurta, R.P.; Meijer, J.-M.; Williams, G.; Zhu, D.; Feng, Y.; Sikorski, M.; et al. Ultrafast melting of the colloidal crystals. 2017, in preparation. 
26. Sulyanova, E.A.; Shabalin, A.; Zozulya, A.V.; Meijer, J.M.; Dzhigaev, D.; Gorobtsov, O.; Kurta, R.P.; Lazarev, S.; Lorenz, U.; Singer, A.; et al. Structural evolution of colloidal crystal films in the process of melting revealed by Bragg peak analysis. Langmuir 2015, 31, 5274-5283. [CrossRef] [PubMed]

27. Gorobtsov, O.Y.; Mukharamova, N.; Lazarev, S.; Chollet, M.; Kurta, R.P.; Meijer, J.-M.; Williams, G.; Zhu, D.; Feng, Y.; Sikorski, M.; et al. Intensity interferometry on crystal diffraction patterns at hard X-ray free electron laser. 2017, in preparation.

28. Hazewinkel, M. Encyclopedia of Mathematics: An Updated and Annotated Translation of the Soviet Mathematical Encyclopedia; Springer: Berlin, Germany, 2013; Volume 2.

29. Lamb, H. On the vibrations of an elastic sphere. Proc. Lond. Math. Soc. 1881, 1, 189-212. [CrossRef]

30. Saviot, L. Vibrational Eigenmodes of an Isotropic Sphere. Available online: http://lucien.saviot.free.fr/ lamb/index.en.html (accessed on 30 March 2017).

31. Mark, J.E. Polymer Data Handbook; Oxford University Press: Oxford, UK, 2009; p. 834.

32. Tas, G; Maris, H.J. Electron diffusion in metals studied by picosecond ultrasonics. Phys. Rev. B 1994, 49, 15046. [CrossRef]

33. Huber, P.; Bunk, O.; Pietsch, U.; Textor, M.; Geue, T. Grazing Incidence Small Angle X-ray Scattering on Colloidal Crystals. J. Phys. Chem. B 2010, 114, 12473-12479. [CrossRef] [PubMed]

34. Zozulya, A.; Zaluzhnyy, I; Lazarev, S.; Meijer, J.-M.; Mukharamova, N.; Shabalin, A.; Kurta, R.P.; Sprung, M.; Petukhov, A.V.; Vartanyants, I.A. Temperature-driven rearrangement of colloidal crystal domains studied in situ by GISAXS. 2017, in preparation.

(C) 2017 by the authors. Licensee MDPI, Basel, Switzerland. This article is an open access article distributed under the terms and conditions of the Creative Commons Attribution (CC BY) license (http:/ / creativecommons.org/licenses/by/4.0/). 